\title{
Complete genome sequence of Kocuria rhizophila BT304, isolated from the small intestine of castrated beef cattle
}

\author{
Tae Woong Whon ${ }^{\dagger}$, Hyun Sik Kim ${ }^{\dagger}$ and Jin-Woo Bae*
}

\begin{abstract}
Background: Members of the species Kocuria rhizophila, belonging to the family Micrococcaceae in the phylum Actinobacteria, have been isolated from a wide variety of natural sources, such as soil, freshwater, fish gut, and clinical specimens. K. rhizophila is important from an industrial viewpoint, because the bacterium grows rapidly with high cell density and exhibits robustness at various growth conditions. However, the bacterium is an opportunistic pathogen involved in human infections. Here, we sequenced and analyzed the genome of the K. rhizophila strain BT304, isolated from the small intestine of adult castrated beef cattle.

Results: The genome of K. rhizophila BT304 consisted of a single circular chromosome of 2,763,150 bp with a GC content of $71.2 \%$. The genome contained 2359 coding sequences, 51 tRNA genes, and 9 rRNA genes. Sequence annotations with the RAST server revealed many genes related to amino acid, carbohydrate, and protein metabolism. Moreover, the genome contained genes related to branched chain amino acid biosynthesis and degradation. Analysis of the OrthoANI values revealed that the genome has high similarity (>97.8\%) with other K. rhizophila strains, such as DC2201, FDAARGOS 302, and G2. Comparative genomic analysis further revealed that the antibiotic properties of $K$. rhizophila vary among the strains.
\end{abstract}

Conclusion: The relatively small number of virulence-related genes and the great potential in production of host available nutrients suggest potential application of the BT304 strain as a probiotic in breeding beef cattle.

Keywords: Kocuria rhizophila BT304, Complete genome sequence, Bovine small intestine, Branched chain amino acids

\section{Background}

Kocuria rhizophila, a coccoid, Gram-positive, and spherical bacterium, was first identified by Kovacs et al. [1]. It belongs to the family Micrococcaceae in the phylum Actinobacteria, and has been isolated from a wide variety of natural sources such as soil [2], freshwater [3], and fish gut [4]. Considering its relatively small genome size among the order Actinomycetales, it is surprising that each $K$. rhizophila strain is highly adapted to its ecological niche. K. rhizophila is important from both ecological

\footnotetext{
*Correspondence: baejw@khu.ac.kr

†Tae Woong Whon and Hyun Sik Kim contributed equally to this work Department of Biology and Department of Life and Nanopharmaceutical Sciences, Kyung Hee University, 26 Kyungheedae-ro, Dongdaemun-gu, Seoul 02447, Republic of Korea
}

and industrial viewpoints, because the bacterium is tolerant to a wide range of organic solvents and capable of growing robustly in various conditions [2, 5]. More recently, attention has been drawn to clinical aspects of $K$. rhizophila. The clinical strains were isolated from blood samples of children with illness [6, 7], suggesting that the bacterium is an opportunistic pathogen involved in human infections. To date, nine strains of K. rhizophila are sequenced, with only two genomes completed. Here, we report the complete genome sequence of $K$. rhizophila BT304, isolated from the small intestine of castrated beef cattle. Genomic evaluation and comparative genomics may improve our understanding of the virulence factors and gut-associated symbiotic potential of K. rhizophila, 
and hence its suitability for probiotic application in the livestock industry.

\section{Methods}

\section{Strain isolation and DNA extraction}

Kocuria rhizophila BT304 was isolated from the small intestine of adult castrated beef cattle. The luminal content of the ileum was collected from a local slaughterhouse (Gunwi-Gun, South Korea). The isolate was cultivated on brain heart infusion (BHI) agar (Becton-Dickinson) in aerobic conditions at $37{ }^{\circ} \mathrm{C}$ for $24 \mathrm{~h}$. Genomic DNA of the isolate was extracted using the MG Genomic DNA Purification Kit (MGmed) according to the manufacturer's instruction. Total DNA was subjected to quality control by electrophoresis on a $1 \%$ agarose gel and quantified by a NanoDrop spectrophotometer (Thermo Scientific) and a Qubit fluorometer (Thermo Scientific).

\section{Whole genome sequencing, assembly, and gene annotation}

The whole genome sequencing of $K$. rhizophila BT304 was performed using PacBio RS II (20-kb SMRTbell ${ }^{\mathrm{TM}}$ templates) and Illumina HiSeq4000 (TruSeq DNA PCRFree 350 -bp library) strategies. For obtaining 20-kb library, genomic DNA was sheared with g-TUBE (Covaris) and purified by AMPure PB magnetic beads (Beckman Coulter). The sequencing library for Illumina HiSeq4000 was prepared by random fragmentation of the DNA sample, followed by $5^{\prime}$ and $3^{\prime}$ adapter ligation. After filtering, we obtained 165,979 sub-reads with mean length of 8036-bp from the PacBio system. From the Illumina data set $(34,363,358$ raw reads), we obtained 12,131,322 filtered reads (quality score over $20(\mathrm{Q} 20)=99.12 \%)$. The reads were assembled using RS HGAP Assembly version 3.0 and polished with Quiver. The assembly of $K$. rhizophila BT304 was annotated using the RAST prokaryotic genome annotation server (http:// rast.nmpdr.org/) [8], and the Position-Specific Iterative BLAST (PSI-BLAST) against the eggNOG version 4.5 database [9]. RNAmmer 1.2 [10] and tRNAscan-SE 1.21 [11] were used to identify rRNA and tRNA sequences, respectively. Prophage insert regions were detected with an on-line phage search tool, PHASTER [12].

\section{Phylogenetic analysis}

We constructed a phylogenetic tree based on 16S rRNA gene sequences. Using the multiple sequence alignment program CLUSTAL W [13], the 16S rRNA gene sequences of the BT304 and related species were aligned. Phylogenetic trees were constructed using the following algorithms: neighbor-joining [14], maximum-parsimony
[15] and maximum-likelihood [16] algorithms based on 1000 bootstrap replications in MEGA version 7 [17].

\section{Comparative genomic analysis}

We performed comparative genomic analysis on nine $K$. rhizophila strains: DC2201, FDAARGOS 302, G2, D2, 14ASP, P7-4, TPW45, RF, and UMD0131. The reference genome sequences were downloaded from the NCBI genome database (http://www.ncbi.nlm.nih.gov/genom e/). Average nucleotide identity (ANI) based on USEARCH (OrthoANIu) was used to assess overall similarity between two genome sequences [18]. Functional genes in each genome were predicted and annotated using the SEED subsystem in the RAST server. For whole genome comparison, the genome of $K$. rhizophila BT304 and other $K$. rhizophila genomes were aligned using the progressive MAUVE algorithm in the MAUVE multiple genome alignment software 2.4.0 [19].

\section{Quality assurance}

A single colony of the $K$. rhizophila strain BT304 was repeatedly transferred to fresh BHI medium to obtain pure cultures. Before DNA extraction, the identity of the strain was verified through $16 \mathrm{~S}$ rRNA gene sequencing. After the genome sequence was obtained, we confirmed the identity of the strain through a BLAST search of the $16 \mathrm{~S}$ rRNA gene found in the genome.

\section{Results and discussion}

\section{General features}

The genome coverage of the K. rhizophila strain BT304 was 329 fold, and the complete genome sequence consisted of a single circular chromosome of 2,763,150 bp. The genome contained 2359 coding sequences, 51 tRNA genes, and 9 rRNA genes. The circular map of the genome is displayed in Fig. 1, and detailed genomic features are listed in Table 1. GC content of the complete genome was $71.2 \%$. The GC content and size of the BT304 chromosome was similar to those of other $K$. rhizophila strains, including $K$. rhizophila DC2201 (2,697,540 bp, 71.2\% GC) [2], K. rhizophila P7-4 (2,820,331 bp, $70.5 \%$ GC) [4], and K. rhizophila TPW45 (2,701,701 bp, 70.6\% GC) [3] (Table 2). The results of RAST annotation showed that the genome contained 2427 coding sequences with 55 RNA genes. In the SEED subsystem distribution, "amino acids and derivatives" (345 ORFs), "carbohydrates" (252 ORFs), "protein metabolism” (206 ORFs), and "cofactors, vitamins, prosthetic groups, pigments" (206 ORFs) were the most abundant categories (Fig. 2). Similar to the RAST annotation results, annotation of the BT304 genome using the eggNOG database revealed "amino acid transport and metabolism" (203 ORFs) as the most abundant category, followed by "replication, 


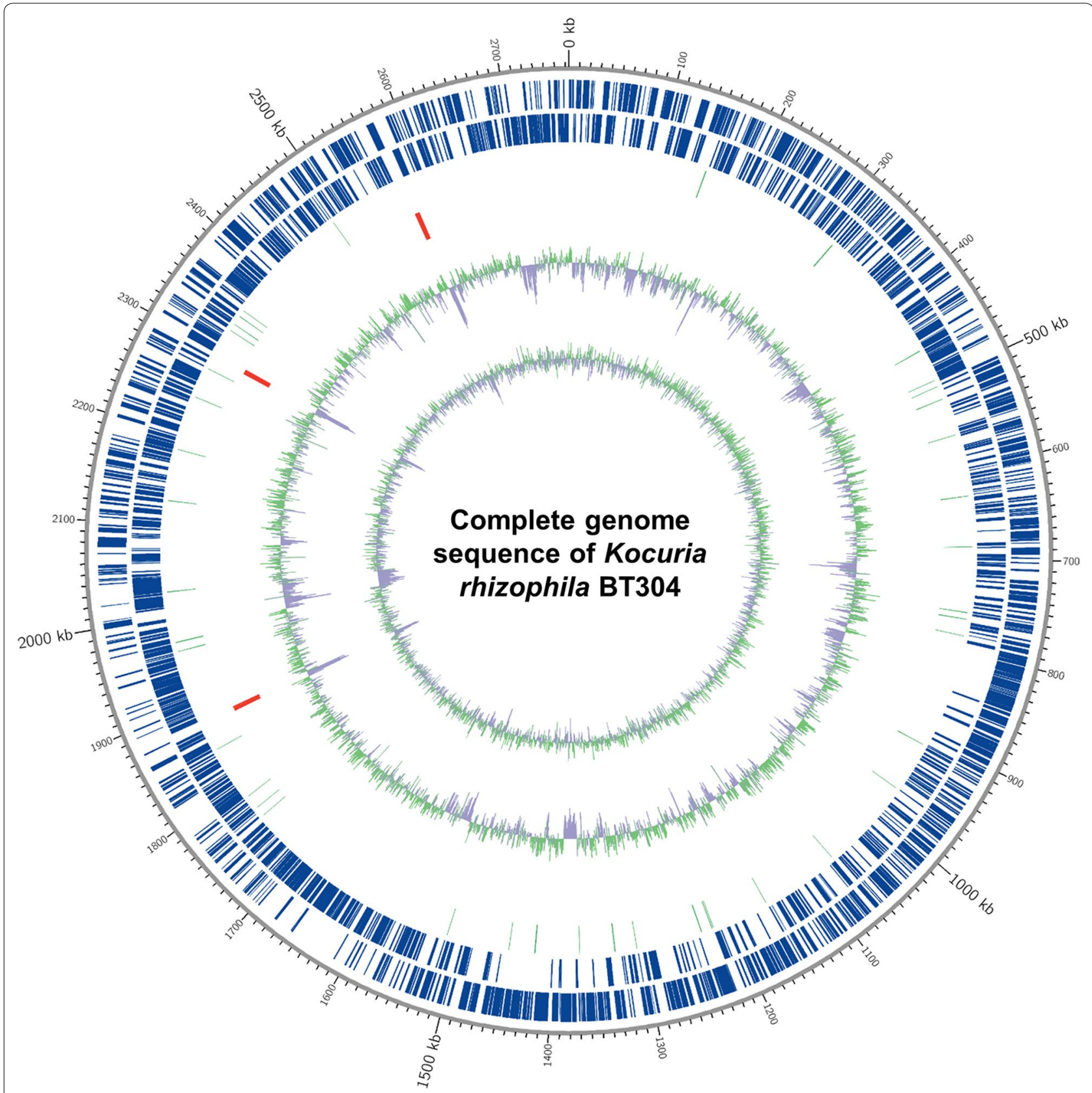

Fig. 1 Graphic circular map of the Kocuria rhizophila BT304 genome. CDSs on the forward strand and CDSs on the reverse strand are indicated from the outer fringe to the center. Inner circles represent the tRNAs (light blue), rRNAs (red), GC content, and GC skew

recombination and repair" (148 ORFs), and "translation, ribosomal structure and biogenesis" (145 ORFs) (Additional file 1: Table S1).

\section{Comparative genomic analysis}

Based on 16S rRNA gene sequences, we constructed a phylogenetic tree to identify the phylogeny of the K. rhizophila strain BT304 within the family Micrococcaceae
(Additional file 1: Figure S1). We compared the genome of $K$. rhizophila BT304 with those of nine other $K$. rhizophila strains. Analysis of the OrthoANI values revealed that the genome of strain BT304 is closest to that of $K$. rhizophila DC2201 (98.92\% OrthoANI), followed by FDAARGOS 302 (98.80\%), and G2 (97.89\%) (Table 2). Between two genomes, an ANI value higher than 95-96\% is regarded as the same species [20]. Accordingly, the 
Table 1 Complete genome features of Kocuria rhizophila BT304

\begin{tabular}{ll}
\hline Item & Values \\
\hline Sequencing platforms & $\begin{array}{c}\text { PacBio RS II } \\
\text { and Illumina } \\
\text { HiSeq }\end{array}$ \\
Topology & Circular \\
Genome size (bp) & $2,763,150$ \\
Genome coverage (fold) & 329 \\
DNA G + C (\%) & 71.2 \\
tRNA genes & 51 \\
rRNA genes & 9 \\
Coding sequences & 2359 \\
\hline
\end{tabular}

strain BT304 was confirmed to be a species of $K$. rhizophila. Interestingly, however, the genome of ВT304 showed much less genomic relatedness with several other K. rhizophila strains: D2 (88.14\%), 14ASP (87.97\%), P7-4 (87.96\%), TPW45 (87.92\%), RF (87.90\%), and UMD0131 $(87.83 \%)$. We next conducted whole genome comparison of the genome of $K$. rhizophila BT304 with other publically available $K$. rhizophila genomes based on the MAUVE multiple genome alignment. The locally collinear blocks between the BT304 and DC2201 genomes were highly homologous, whereas considerable differences in genomic regions were observed between the BT304 and other K. rhizophila genomes (Fig. 3). These results imply that each $K$. rhizophila strain can adapt to its respective ecological niche by gaining and/or losing genomic elements.

\section{Virulence-related factors}

Because several strains belonging to the species $K$. rhizophila have been isolated from clinical specimens (i.e., human blood) [6, 7], we evaluated virulence-related factors from the genome of strain BT304 and other publically available $K$. rhizophila genomes. Based on comparison with the SEED database, we found 28-46 CDSs annotated as the "virulence, disease and defense" category from the K. rhizophila genomes (Table 2). The BT304 strain possessed the lowest number of virulencerelated genes (28 CDSs), whereas the 14ASP strain (isolated from soil) contained the highest number of virulence-related genes (46 CDSs). To determine whether a degree of virulence-related factors observed from the strain BT304 is suitable for probiotic applications, we accessed the number of virulence-related genes in genomes of eight commercially available probiotic strains [21]. The probiotic strains mainly composed of the genera Bifidobacterium and Lactobacillus contained 22-47 CDSs assigned to the "virulence, disease and defense" category (Additional file 1: Table S2).
We next assessed antibiotic resistant potential in the K. rhizophila genomes. Genes related to the sub-category "resistance to fluoroquinolones" were found in the genomes of all K. rhizophila strains. Several strains (e.g., BT304, DC2201, FDAARGOS 302, G2, and RF) contained genes related to "resistance to vancomycin". Only the UMB0131 strain isolated from human urine possessed genes related to "multidrug resistance efflux pumps". Collectively, our comparative analysis suggested that the antibiotic properties of $K$. rhizophila vary among the strains.

\section{Prophage insertion}

To identify prophage contamination in the genome of $K$. rhizophila BT304, we conducted PHAST phage search analysis. The genome contained four incomplete prophages at positions 876,510-884,942, 1,118,199$1,125,394, \quad 1,367,325-1,389,936$, and 2,021,0032,029,604 bp (Additional file 1: Table S3). PHAST analysis in other $K$. rhizophila strains further revealed that the number of prophages varies among the strains. Strains with an ANI of over 97\% with the strain BT304 were predicted to have 1-3 incomplete prophages, whereas the remaining strains contained no prophage. The strain P7-4, isolated from the fish gut, was the only exception, containing one incomplete prophage.

\section{Branched chain amino acid metabolism}

The BT304 strain was isolated from the bovine small intestine where dietary nutrients (e.g., amino acids, lipids, and carbohydrates) are metabolized by abundant microbiota [22, 23]. As described above, the BT304 strain possessed a relatively small genome, but many genes related to amino acid metabolism (Fig. 2 and Additional file 1: Table S1). Based on comparison with the SEED database, we found a relatively similar number of genes (318-356 CDSs) annotated as the "amino acid and derivatives" category across the $K$. rhizophila genomes (Table 2), suggesting that K. rhizophila can utilize dietary amino acids, and might be an indigenous member of the small intestinal gut microbiota in beef cattle.

In mammals, including human and mouse, branched chain amino acids (BCAAs) are responsible for lipogenesis in adipocytes [24] and lipid accumulation in skeletal muscles [25]. Therefore, accumulation of BCAAs is regarded as a key risk factor leading to obesity. However, these metabolic aspects of BCAAs would be beneficial in economical feeding of breeding animals. Annotating the genome of BT304 using the SEED database revealed genes related to both BCAA biosynthesis (20 CDSs) and degradation (52 CDSs) (Table 2). 


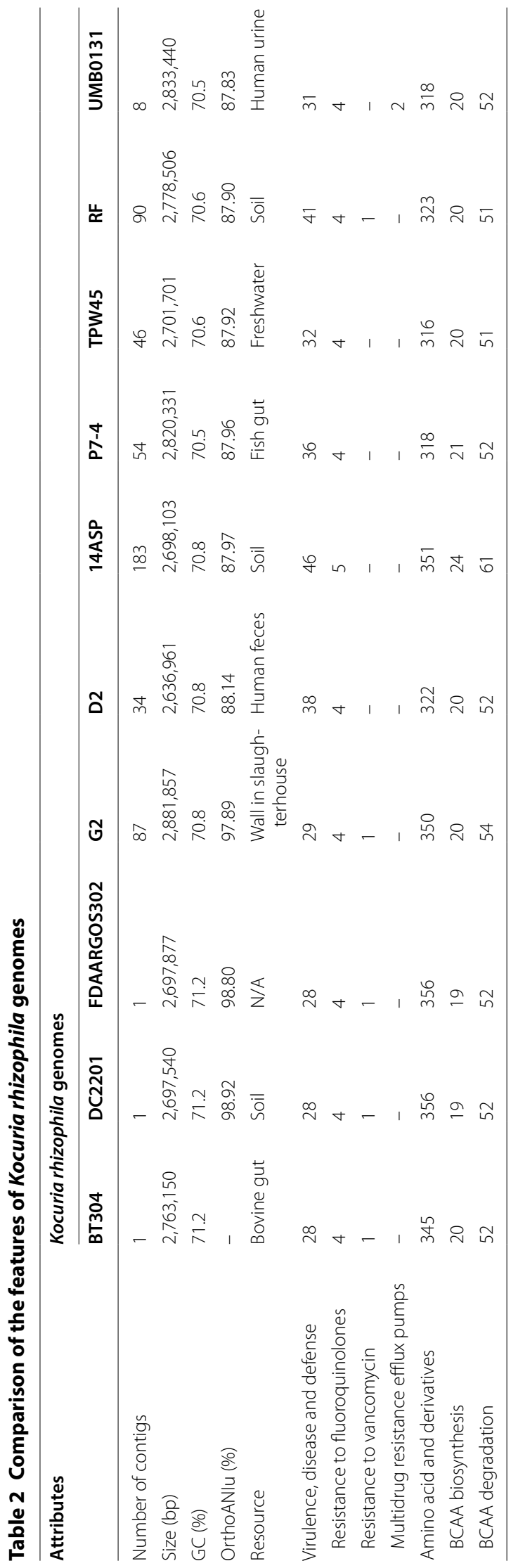




\section{Subsystem Category Distribution}

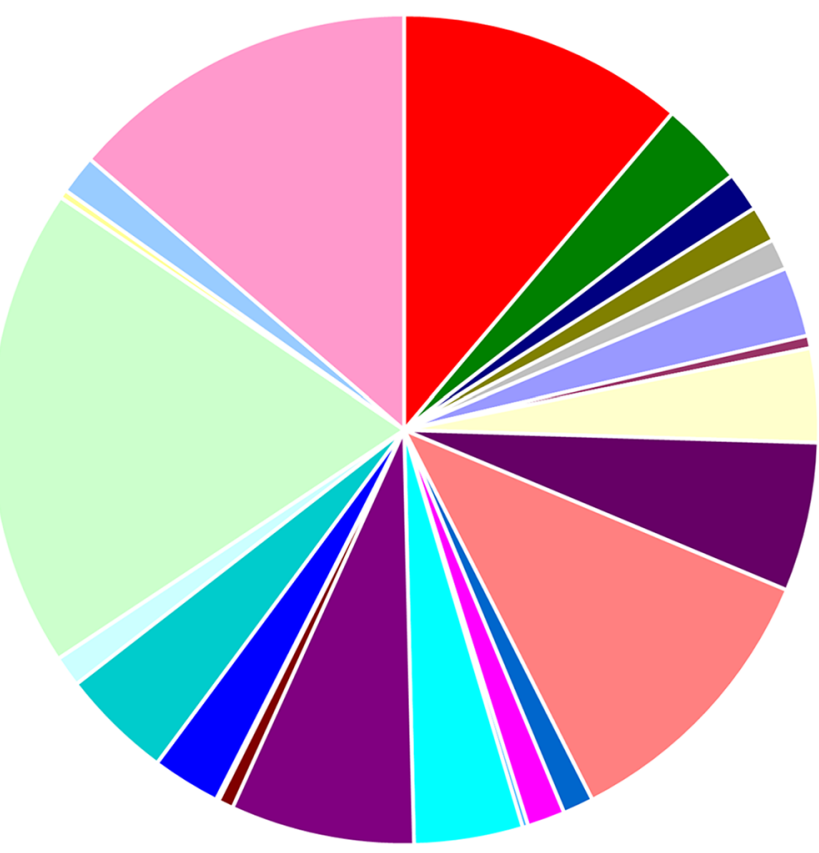

- Cofactors, Vitamins, Prosthetic Groups, Pigments (206)

- Cell Wall and Capsule (61)

- Virulence, Disease and Defense (28)

- Potassium metabolism (26)

- Photosynthesis (0)

- Miscellaneous (22)

- Phages, Prophages, Transposable elements, Plasmids (0)

- Membrane Transport (50)

- Iron acquisition and metabolism (9)

RNA Metabolism (67)

- Nucleosides and Nucleotides (107)

- Protein Metabolism (206)

- Cell Division and Cell Cycle (22)

- Motility and chemotaxis (0)

- Regulation and Cell signaling (27)

- Secondary Metabolism (4)

= DNA Metabolism (78)

- Fatty Acids, Lipids, and Isoprenoids (132)

- Nitrogen Metabolism (11)

- Dormancy and Sporulation (2)

- Respiration (49)

- Stress Response (80)

Metabolism of Aromatic Compounds (22)

Amino Acids and Derivatives (345)

Sulfur Metabolism (6)

Phosphorus Metabolism (28)

Carbohydrates (252)

Fig. 2 Analysis of annotated genes for the Kocuria rhizophila BT304 genome based on the SEED database. Of 2427 CDSs predicted by the RAST server, the subsystem coverage was $48 \%$, contributing 361 subsystems

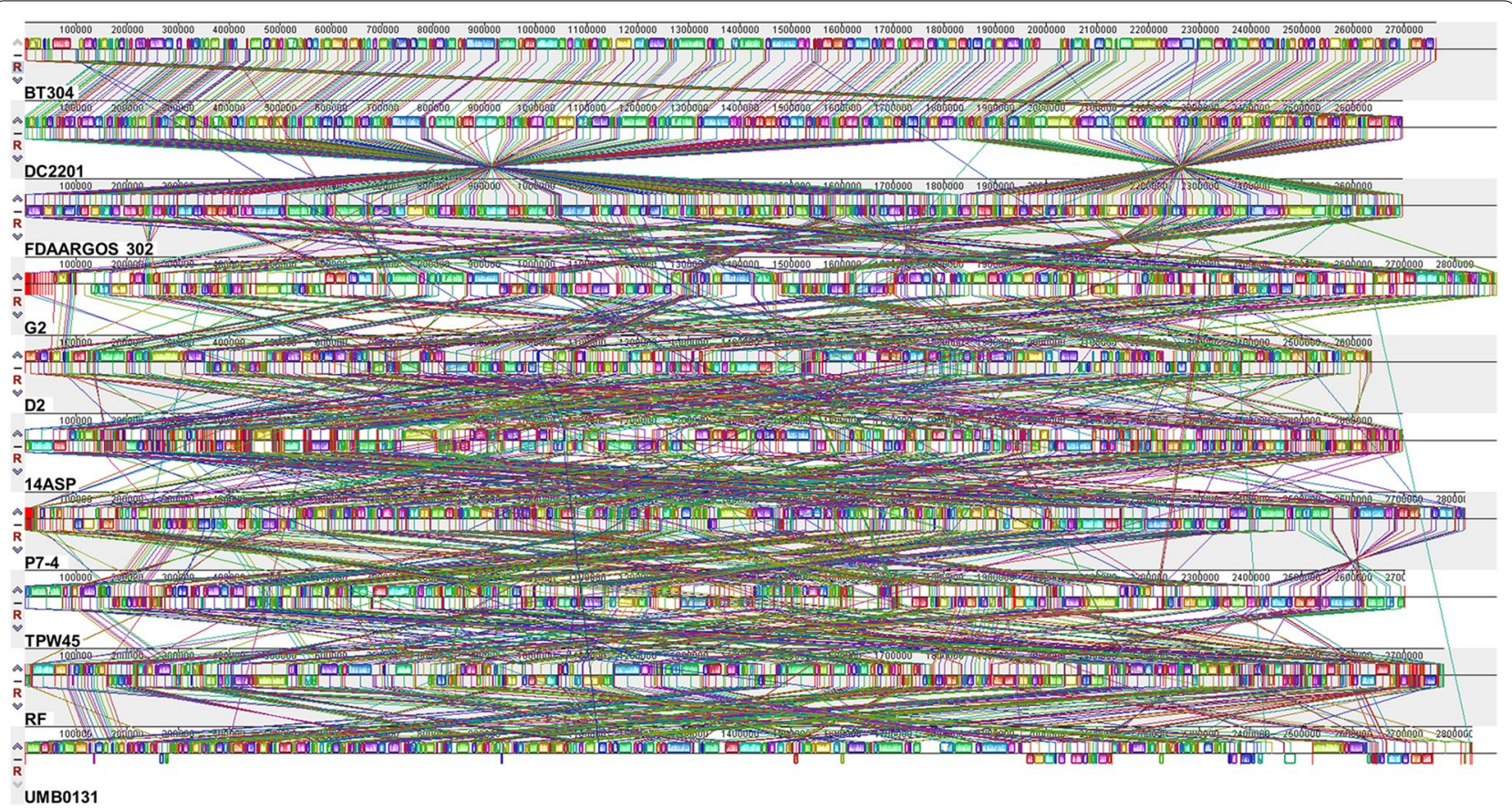

Fig. 3 MAUVE alignment of the genome of Kocuria rhizophila BT304 and other K. rhizophila genomes. The locally collinear blocks shown with identical colors denote highly homologous regions. The genomes were drawn to scale based on the genome of K. rhizophila BT304 
Comparative genomic analysis showed that other reference $K$. rhizophila genomes also contain more than 70 genes related to BCAA metabolism.

\section{Conclusions}

We described the complete genome sequence of $K$. rhizophila BT304, isolated from the small intestine of castrated beef cattle. Our genomic analysis suggests that the relatively small number of virulence-related genes in combination with the potential for the production of host available nutrients make the K. rhizophila strain BT304 a probiotic candidate in breeding beef cattle.

\section{Additional file}

Additional file 1: Figure S1. Phylogenetic tree based on $16 \mathrm{~S}$ rRNA gene sequences, reconstructed with the neighbour-joining $(\mathrm{NJ})$, maximumparsimony (MP) and maximum-likelihood (ML) algorithms, indicating the taxonomic positions of strain BT304 and close relatives in the family Micrococcaceae. Table S1. Analysis of annotated genes for the Kocuria rhizophila BT304 genome based on the eggNOG database. Table S2. Comparison of the virulence related factors in commercially available probiotics. Table S3. Phage sequences found in Kocuria rhizophila genomes.

\section{Abbreviations}

ANI: average nucleotide identity; CDS: coding DNA sequence; ORF: open reading frame; BCAA: branched chain amino acid.

\section{Authors' contributions}

JWB and TWW designed the experiments. TWW and HSK performed the experiments and analyzed the data. TWW, HSK, and JWB wrote the manuscript. All authors read and approved the final manuscript.

\section{Acknowledgements}

We thank the farm owners, veterinarians, and slaughterhouse staff for helping with sample collection. We especially thank Hong Gil Kim (President of the National Hanwoo Association) for advising on the sampling strategy and for helpful discussions.

\section{Competing interests}

The authors declare that they have no competing interests.

\section{Availability of data and materials}

The complete genome data of Kocuria rhizophila BT304 has been deposited into GenBank under Accession Number CP030039.

\section{Consent for publication}

Not applicable.

\section{Ethics approval and consent to participate}

The study protocol was approved by the Institutional Review Boards of Kyung Hee University [KHUASP, (SE)-17-026], and the experiments were performed in agreement with the ARRIVE guidelines [26].

\section{Funding}

This study was supported by the Korea Institute of Planning and Evaluation for Technology in Food, Agriculture, Forestry (IPET) through the Agricultural
Microbiome R\&D Program, funded by the Ministry of Agriculture, Food and Rural Affairs (MAFRA) (Grant Number: 918011-4), and the National Research Foundation of Korea (NRF) grant funded by the Korea government (MSIT) (Grant Number: NRF-2018R1A5A1025077).

\section{Publisher's Note}

Springer Nature remains neutral with regard to jurisdictional claims in published maps and institutional affiliations.

Received: 16 July 2018 Accepted: 24 September 2018

Published online: 27 September 2018

\section{References}

1. Kovacs G, Burghardt J, Pradella S, Schumann P, Stackebrandt E, Marialigeti K. Kocuria palustris sp. nov. and Kocuria rhizophila sp. nov., isolated from the rhizoplane of the narrow-leaved cattail (Typha angustifolia). Int I Syst Bacteriol. 1999;49(Pt 1):167-73.

2. Takarada H, Sekine M, Kosugi H, Matsuo Y, Fujisawa T, Omata S, Kishi E, Shimizu A, Tsukatani N, Tanikawa S, et al. Complete genome sequence of the soil actinomycete Kocuria rhizophila. J Bacteriol. 2008:190:4139-46.

3. Adrian TG, Tan PW, Chen JW, Yin WF, Chan KG. Draft genome sequence of Kocuria rhizophila strain TPW45, an actinobacterium isolated from freshwater. J Genomics. 2016;4:16-8.

4. Kim WJ, Kim YO, Kim DS, Choi SH, Kim DW, Lee JS, Kong HJ, Nam BH, Kim BS, Lee SJ, et al. Draft genome sequence of Kocuria rhizophila P7-4. J Bacteriol. 2011;193:4286-7.

5. Fujita K, Hagishita T, Kurita S, Kawakura Y, Kobayashi Y, Matsuyama A, Iwahashi H. The cell structural properties of Kocuria rhizophila for aliphatic alcohol exposure. Enzyme Microb Technol. 2006;39:511-8.

6. Becker K, Rutsch F, Uekotter A, Kipp F, Konig J, Marquardt T, Peters G, von Eiff C. Kocuria rhizophila adds to the emerging spectrum of micrococcal species involved in human infections. J Clin Microbiol. 2008;46:3537-9.

7. Moissenet D, Becker K, Merens A, Ferroni A, Dubern B, Vu-Thien H. Persistent bloodstream infection with Kocuria rhizophila related to a damaged central catheter. J Clin Microbiol. 2012:50:1495-8.

8. Aziz RK, Bartels D, Best AA, DeJongh M, Disz T, Edwards RA, Formsma K, Gerdes S, Glass EM, Kubal M, et al. The RAST Server: rapid annotations using subsystems technology. BMC Genomics. 2008;9:75.

9. Huerta-Cepas J, Szklarczyk D, Forslund K, Cook H, Heller D, Walter MC, Rattei T, Mende DR, Sunagawa S, Kuhn M, et al. eggNOG 4.5: a hierarchical orthology framework with improved functional annotations for eukaryotic, prokaryotic and viral sequences. Nucleic Acids Res. 2016:44:D286-93.

10. Lagesen K, Hallin P, Rodland EA, Staerfeldt HH, Rognes T, Ussery DW. RNAmmer: consistent and rapid annotation of ribosomal RNA genes. Nucleic Acids Res. 2007;35:3100-8.

11. Lowe TM, Eddy SR. tRNAscan-SE: a program for improved detection of transfer RNA genes in genomic sequence. Nucleic Acids Res. 1997;25:955-64.

12. Arndt D, Grant JR, Marcu A, Sajed T, Pon A, Liang Y, Wishart DS. PHASTER: a better, faster version of the PHAST phage search tool. Nucleic Acids Res. 2016:44:W16-21.

13. Thompson JD, Higgins DG, Gibson TJ. CLUSTAL W: improving the sensitivity of progressive multiple sequence alignment through sequence weighting, position-specific gap penalties and weight matrix choice. Nucleic Acids Res. 1994:22:4673-80

14. Saitou N, Nei M. The neighbor-joining method: a new method for reconstructing phylogenetic trees. Mol Biol Evol. 1987;4:406-25.

15. Kluge AG, Farris JS. Quantitative phyletics and the evolution of anurans. Syst Biol. 1969;18:1-32.

16. Felsenstein J. Evolutionary trees from gene frequencies and quantitative characters: finding maximum likelihood estimates. Evolution. 1981;35:1229-42.

17. Kumar S, Stecher G, Tamura K. MEGA7: molecular evolutionary genetics analysis version 7.0 for bigger datasets. Mol Biol Evol. 2016;33:1870-4.

18. Yoon SH, Ha SM, Lim J, Kwon S, Chun J. A large-scale evaluation of algorithms to calculate average nucleotide identity. Antonie Van Leeuwenhoek. 2017;110:1281-6. 
19. Darling AC, Mau B, Blattner FR, Perna NT. Mauve: multiple alignment of conserved genomic sequence with rearrangements. Genome Res. 2004; 14:1394-403.

20. Lee I, Ouk Kim Y, Park SC, Chun J. OrthoANI: an improved algorithm and software for calculating average nucleotide identity. Int J Syst Evol Microbiol. 2016;66:1100-3.

21. Douillard FP, Mora D, Eijlander RT, Wels M, de Vos WM. Comparative genomic analysis of the multispecies probiotic-marketed product VSL\#3. PLOS ONE. 2018:13:e0192452.

22. Donaldson GP, Lee SM, Mazmanian SK. Gut biogeography of the bacterial microbiota. Nat Rev Microbiol. 2016;14:20-32.

23. Kiela PR, Ghishan FK. Physiology of intestinal absorption and secretion. Best Pract Res Clin Gastroenterol. 2016;30:145-59.
24. Green CR, Wallace M, Divakaruni AS, Phillips SA, Murphy AN, Ciaraldi TP, Metallo CM. Branched-chain amino acid catabolism fuels adipocyte differentiation and lipogenesis. Nat Chem Biol. 2016;12:15-21.

25. Jang C, Oh SF, Wada S, Rowe GC, Liu L, Chan MC, Rhee J, Hoshino A, Kim B, Ibrahim A, et al. A branched-chain amino acid metabolite drives vascular fatty acid transport and causes insulin resistance. Nat Med. 2016;22:421-6.

26. Kilkenny C, Browne WJ, Cuthill IC, Emerson M, Altman DG. Improving bioscience research reporting: the ARRIVE guidelines for reporting animal research. Plos Biol. 2010;8:e1000412.
Ready to submit your research? Choose BMC and benefit from:

- fast, convenient online submission

- thorough peer review by experienced researchers in your field

- rapid publication on acceptance

- support for research data, including large and complex data types

- gold Open Access which fosters wider collaboration and increased citations

- maximum visibility for your research: over $100 \mathrm{M}$ website views per year

At BMC, research is always in progress.

Learn more biomedcentral.com/submissions 